\title{
Managing infertility with the follitropin alfa prefilled pen injector - patient considerations
}

\author{
This article was published in the following Dove Press journal: \\ Therapeutics and Clinical Risk Management \\ 29 June 2015 \\ Number of times this article has been viewed
}

\section{Klaus Bühler \\ Centre for Gynaecological Endocrinology and Reproductive Medicine, Stuttgart, Germany}

Video abstract

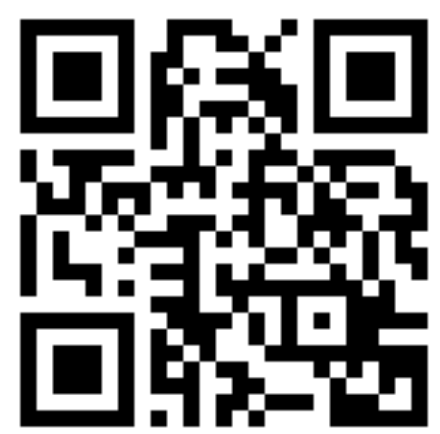

Point your SmartPhone at the code above. If you have a QR code reader the video abstract will appear. Or use: http://youtu.be/F t00Kfgv0i8

Correspondence: Klaus Bühler Centre for Gynaecological Endocrinology and Reproductive Medicine, Friedrichstraße 45, D-70174 Stuttgart, Germany

Email buehler@ivf-zentrum
Abstract: Gonadotropin treatment has been used in fertility treatment since the 1930s. First, preparations coming from animals were injected, then, gonadotropins prepared from the pituitary glands of human cadavers. A great step was achieved with the introduction of human menopausal gonadotropin extracted from the urine of postmenopausal women. When cases of CreutzfeldJacob disease were recognized after the use of human pituitary-derived hormone injections, urinary gonadotropins were increasingly purified and then produced by the use of recombinant DNA technology. Recombinant gonadotropins were characterized by the extreme high specificity and the nearly $100 \%$ purity. This allows for follitropin alfa, the first recombinant-human follicle stimulating hormone (r-hFSH) approved, to be quantified and filled by mass, with a small variance of only $\pm 2 \%$ and no more with a bioassay with a variance of $45 \%$. With recombinant preparations, it is also possible to cover the tremendous growing demand for gonadotropins. Ovarian stimulation has become a self-injecting procedure for the patients. Accurate and easy-to-use injection devices which minimize pain, difficulty, and stress are essential for patient compliance. So, two pen injectors adapted from the well-known insulin pen were introduced in fertility treatment, one as a multiple-use device rechargeable with premixed, prefilled cartridges with r-hFSH (follitropin $\beta$ ) and the other a disposable, prefilled drug delivery system with a liquid formulation of follitropin alfa filled by mass. The efficacy in comparison to the quite more cumbersome handling with ampoules and syringes has been proven very quickly. In several studies, it has been shown that patients had a preference to the prefilled follitropin alfa pen due to the faster preparation and were more confident of accurate dosing. The follitropin alfa (filled by mass $[\mathrm{FbM}]$ ) prefilled pen is a move toward better quality of treatment and also better quality of life for the women within the stressful period of fertility treatment.

Keywords: assisted reproductive technology, recombinant human gonadotropins, urinaryderived gonadotropins, devices for gonadotropin injection, controlled ovarian stimulation, self-injection

\section{Introduction}

Since humankind has existed, it has always been one of the existential problems that not every couple achieves pregnancy at the moment when the couple wants to have a child.

Mostly, normal fertile couples will conceive within 12 months without practicing any kind of contraception. But the definition of infertility by the World Health Organization (WHO), according to the predominantly used demographers, is: "Number of women of reproductive age (15-49 years) at risk of becoming pregnant who report trying unsuccessfully for a pregnancy for two years or more". 1

Even with an exact definition, prevalence rates in infertility are very difficult to identify due to the fact that both females and males are involved. Furthermore, it must be taken into account that within the last decades a reduction in general child-seeking 
as well as a significant increase in age of the couples desiring their first child has been observed. ${ }^{2,3}$ Based on several WHO studies, it can be estimated that approximately 48.5 million couples worldwide were unable to have a child after 5 years. ${ }^{4}$ It can be seen that there is a great difference between primary and secondary infertility: 1.9\% (confidence interval [CI]: 1.7-2.2) and 10.5\% (CI: 9.5-11.7). The highest infertility prevalence is seen in South Asia, sub-Saharan Africa, North Africa/Middle East, Central and Eastern Europe, and Central Asia. ${ }^{4}$ It is estimated that in developing countries one in every four couples is affected by fertility problems, mostly due to sexually transmitted diseases. Only approximately 5\% of infertility can be explained by hereditary abnormalities of the reproductive organs. It is not difficult to understand that since primeval times, often a lot of ritual acts have been performed, especially in women even if the cause for the couples' infertility was attributed to the man. Since modern diagnostic techniques have come into existence, it is easier to determine which partner is more responsible for the infertility. The Department of Health of the United Kingdom published in 2009 the following distribution: female 30\%, male $30 \%$, both $10 \%$, unexplained $25 \%$, and $5 \%$ other reasons. ${ }^{5}$ When in-vitro fertilization (IVF) is performed in $60 \%$ of the cases, the indication for treatment is seen in the women, when intracytoplasmic sperm injection has to be done, $60 \%$ the indication is justified by male fertility disturbances. ${ }^{3}$

\section{History of gonadotropins}

Gonadotropin injections for ovarian stimulation were introduced in modern fertility treatment in the 1930s and were first prepared from different animal sources: pregnant mare serum gonadotrophin and swine and ovine pituitaries extractions. These drugs continued to be used until the early 1960s. But all these animal preparations had to be withdrawn from the market because of the detected antibody formation against natural human pituitary gonadotropin. ${ }^{6}$ Since 1958 , gonadotropins were extracted from pituitary glands of human cadavers human pituitary gonadotropin (hPG). But they had to be withdrawn when cases of iatrogenic Creutzfeld-Jacob disease were detected. A milestone in the gonadotropin treatment was the introduction of the two-step protocol in the 1940s: first, stimulation of follicular growth and then induction of final oocyte maturation and ovulation with human chorionic gonadotropin (hCG) - first extracted from human placentas, then, as today, from urine of pregnant women. ${ }^{6}$ Even 75 years later, this principle is still carried out. The first human menopausal gonadotropin (hMG) preparation, produced by isolation and purification from crude extracts of large urine pools of postmenopausal women, finally resulted in a relatively simple procedure $^{7}$ that was registered in 1950, and in 1963, was introduced into clinical use as "Pergonal 75", a preparation with 75 IU follicle stimulating hormone (FSH) and 75 IU luteinizing hormone ( $\mathrm{LH})$, with tolerated variance of $-20 \%$ to $+25 \%$ measured by a standard bioassay. The first pregnancy with this compound was reported by Lunenfeld. ${ }^{8}$ While in the early hMG preparations $>95 \%$ were impurities, in the highly purified (HP) preparations of today the rate of impurities is only about 30\%. ${ }^{9}$ But during the purification process, natural LH activity disappears so that $>95 \%$ of the biological LH activity in the purified preparations result from spiking with hCG. After the disaster with hPG, every endeavor has been made to purify urinary gonadotropins in order to achieve a greater safety for the patients. FSH HP achieved the highest degree in purity, $>95 \%$. But in all urinary preparations, $\mathrm{u}-\mathrm{hMG}$ and $\mathrm{u}-\mathrm{hCG}$, recently, human prion proteins with all their theoretical risks have been detected. ${ }^{10}$ Therefore, it seems logical that several committees all over the world, like in Australia or UK, have published the recommendation to replace such drugs with recombinant products with likely higher standards of purity and safety. ${ }^{11}$ The first "pure" hFSH, follitropin alfa - Gonal-F ${ }^{\circledR}$ (Merck Senoro, Darmstadt, Germany), produced in 1988 by recombinant DNA technology, using a cell culture system with Chinese hamster ovary cells, was licensed in Europe in 1996. A quite similar recombinant-human follicle stimulating hormone (r-hFSH) (follitropin $\beta$ - Puregon ${ }^{\circledR} /$ Follistim $^{\circledR}$ [Merck Sharpe \& Dohme, Kenilworth, NJ, USA]) was approved 1 year later. ${ }^{12}$ During the ensuing period, the efficacy of r-hFSH was proven by countless studies. ${ }^{13}$ Due to the higher bioavailability of the recombinant $\mathrm{FSH}$, a higher number of oocytes - which represents a robust surrogate outcome for clinical success - is retrieved in IVF treatment with a lower dose and lower rate of ovarian hyperstimulation syndrome (OHSS) as compared to urinary gonadotropins (Figure 1). ${ }^{14}$ Even when in randomized controlled studies no statistically significant differences between $\mathrm{hMG}$ and $\mathrm{r}-\mathrm{hFSH}$ with regard to live birth rate could be found, there exists clinical evidence of differences between hMG and r-hFSH concerning dose variability and purity. ${ }^{15-18}$

In the following years, recombinant $\mathrm{LH}$ and recombinant hCG, the latter since many years is also available in a pen for self-injection, were available for fertility treatment (Figure 2). In addition, a fixed combination of r-hFSH and r-hLH in a 2:1 ratio (150 IU r-hFSH and 75 IU r-hLH; Pergoveris $^{\circledR}$ [Merck Senoro]) was introduced on the market. A long-acting formulation of recombinant FSH, corifollitropin alfa, has also become available. 


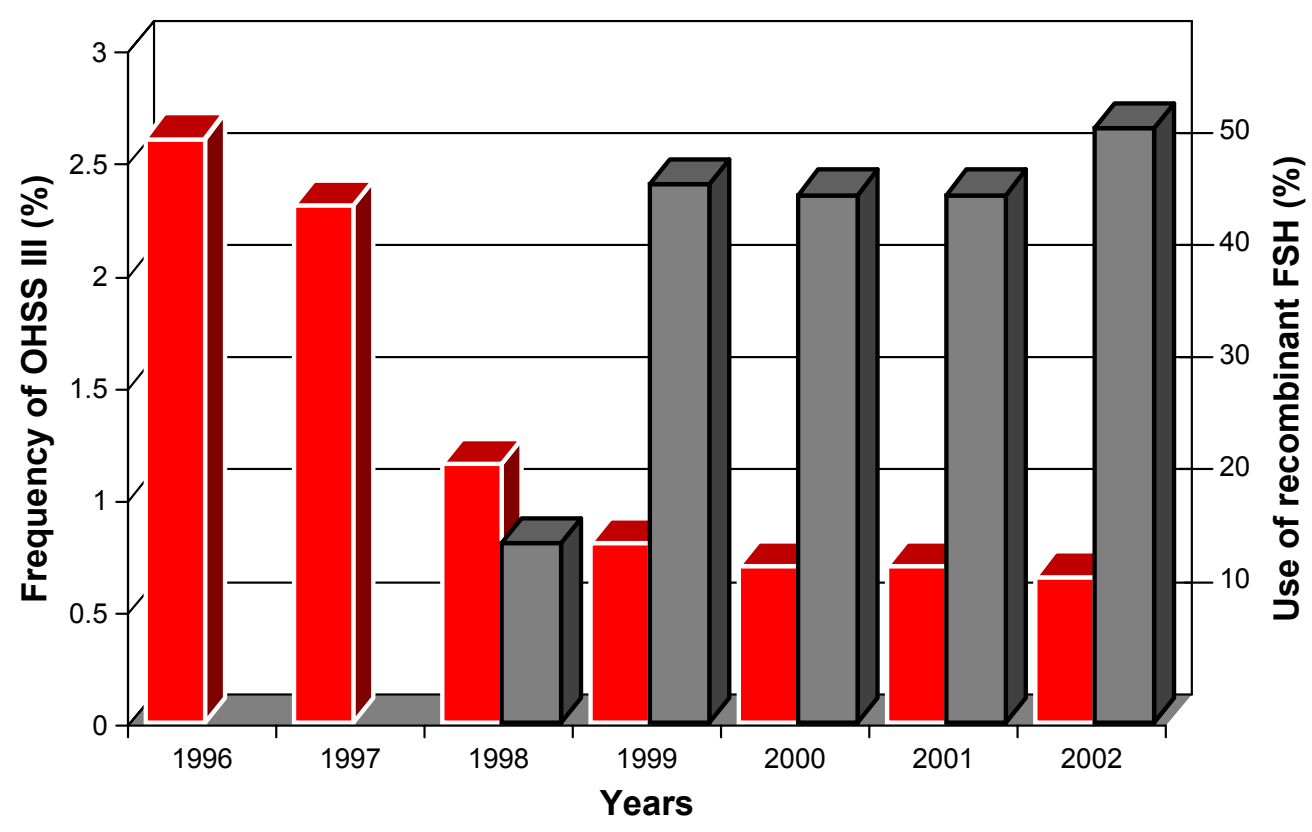

Figure I Relation of frequency of OHSS and the use of recombinant $\mathrm{hFSH}$.

Notes: r-hFSH was introduced into the market in Germany in 1998. Data from the German IVF-Registry - D.I.R; www.deutsches-ivf-register.de. ${ }^{33}$

Abbreviations: $\mathrm{FSH}$, follicle stimulating hormone; OHSS, ovarian hyperstimulation syndrome; $r$-hFSH, recombinant-human follicle stimulating hormone.

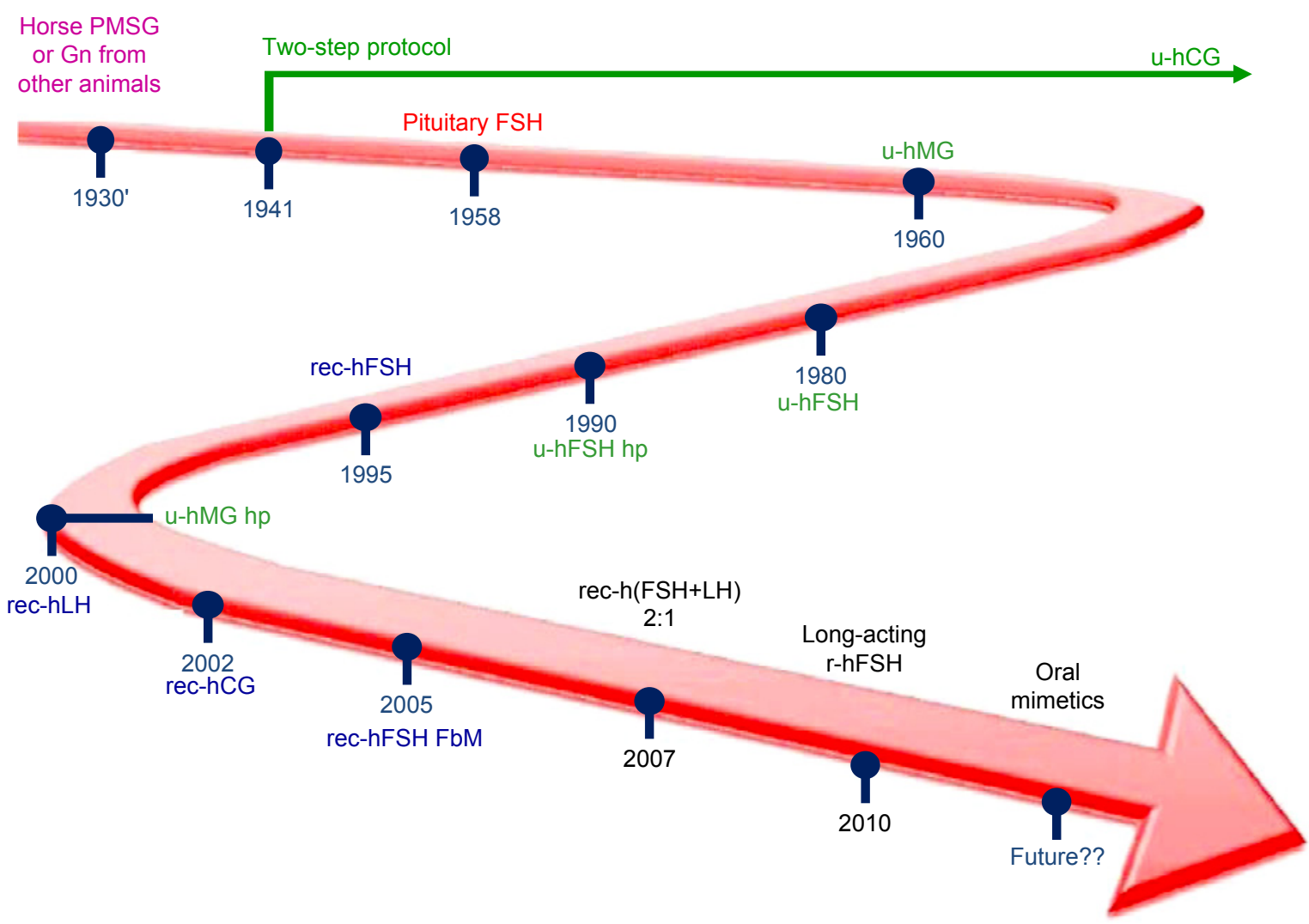

Figure 2 Significant milestones in gonadotropin development.

Notes: The more the gonadotropins are purified, the higher is the specific activity and consistency, providing a greater safety for the patients. In green: urinary products still on the market; in blue: recombinant gonadotropins up to filled by mass; in black: newer recombinant preparations and the future.

Abbreviations: FbM, filled by mass; hp, highly purified; Gn, gonadotropin; rec-h(FSH+LH), fixed combination of recombinant FSH and recombinant LH in the ratio 2:I: Pergoveris ${ }^{\circledR}$ (Merck Senoro, Darmstadt, Germany); FSH, follicle stimulating hormone; LH, leutinizing hormone; hMG, urinary human menopausal gonadotropin; hCG, human chorionic gonadotropin; PMSG, pregnant mare serum gonadotropin; rec, recombinant. 
But, a further big step forward to greater accuracy and safety of gonadotropin ovarian stimulation in reproductive medicine has been follitropin alfa FbM. Due to its high specificity and purity, it can be filled by mass with a very small variance of only $\pm 2 \%$ and quantification is no more performed, as in the urinary gonadotropins or other r-hFSH preparations, by the more imprecise bioassays (as discussed earlier). ${ }^{19}$ Only with such a precise dosing, individualized controlled ovarian stimulation (COS) is possible. It is important for the patient that an injected dose of eg $225 \mathrm{IU}$ is really limited between $220.5 \mathrm{IU}$ and $229.5 \mathrm{IU}( \pm 2 \%)$ and not between $180 \mathrm{IU}$ and $282 \mathrm{IU}(-20 \% /+25 \%)$ as it allowed in all gonadotropins filled by bioassay. It has been shown that calibrating by the amount of mass provides a quite better batch-to-batch consistency that also yields very positive clinical effects. This preparation improves the ovarian response consistency, makes it more predictable, yields better pregnancy rates and less treatment days, and reduces the risks, eg, of OHSS or cycle cancellation. ${ }^{20-22}$

\section{Pen injector}

Gonadotropin treatment has become more and more a selfinjection procedure for the patients. So, it was a logical step to develop pen devices as they were known to have worked for a long time as in the treatment of diabetes with insulin.

There is a great difference between infertility patients and patients using such devices in chronic illness. Unlike other patients, infertility patients take their medication during one treatment cycle, normally only for a short period of 12-25 days, and value every single drop of medication. One reason for this is that in a very great number of countries they have to pay by themselves for these drugs. Very often, these patients are extremely anxious that they deliver the medication correctly. During the treatment cycle, most of the patients are very stressed about giving themselves injections and are not thinking about anything else other than taking the right drugs at the right time. In cases of unsuccessful oocyte retrieval, technical problems such as errors in gonadotropin administration has been described. ${ }^{23}$ Approximately 15.2\% of patients made errors when reconstituting and administering $\mathrm{hCG}$ as a lyophilized powder with the conventional syringes, which influenced pregnancy success. ${ }^{24}$ Overall, the whole infertility issue is emotionally hard for them. For all these reasons, accurate dose delivery is very important, not only from the medical but also the patients' point of view. Accurate and easy-to-use injection devices which minimize pain, difficulty, and stress are essential for patient compliance.
The pen injectors were adapted based on the insulin pen. In comparison with conventional syringes, they have been shown to have a greater acceptance by the diabetes patients. They provide a more accurate, easier, and, what is also very important especially for women when fertility treatment is performed, more discrete injection mode. Their efficacy in comparison to the quite more cumbersome handling with ampoules and syringes has been proven very quickly. ${ }^{25}$ All in all, in two further pilot studies with 171 patients, two follitropin injection pens for subcutaneous self-injection were compared for ease-of-use, safety, and efficacy. One pen is a multiple-use device, which is rechargeable and has premixed, prefilled cartridges with r-hFSH (follitropin $\beta$ ) in different dosages and the other is a disposable, prefilled drug delivery system of multiple and variable doses of a liquid formulation of follitropin alfa filled by mass. Both are delivered with very thin 29-gauge needles for subcutaneous injection, and both had dose increments of 25 and 37.5 IU, respectively, at the time of the study. In the first study $(n=31)$ patients favored the prefilled, disposable device and declared that the preparation was faster and that they were more confident of accurate dosing and had to make fewer dose adjustments. Less time was needed to read the training manual $(67 \% ; 7.1$ vs 12.5 minutes), to prepare the first injection $(63 \% ; 1.9$ vs 3.1 minutes), and to prepare the daily injections ( $69 \%$; 78 vs 132 seconds), and the patients were more confident that the right dose had been administered (on a $1-5$ scale: 1.9 vs 2.2). Approximately $53.3 \%$ preferred the prefilled, nonreturnable pen vs $26.6 \%$ preferring the reusable pen with cartridges. The same result was found in the second study in which a similar questionnaire was used in 140 patients. Both pens were well tolerated. But with the follitropin alfa pen, less adverse events occurred and the risk of OHSS was considered reduced due to the nearly $50 \%$ lower rate of cycle cancellation. Patients also reported less confusion with the use of the prefilled follitropin alfa pen. The follitropin alfa pen was effective for COS, and the acceptance by patients as well nurses was higher for a lot of reasons, including the device storage, device use, and dose preparation. This may lead to improved outcomes. The experience of IVF nurses with both pens was also assessed using a similar questionnaire. Nurses are an integral part and key success factor in training patients on how to correctly self-inject the medication. Most importantly, teaching the patients is much easier and therefore less time consuming for the nurse, which ultimately increases her availability for other tasks in the clinic. ${ }^{26}$

In another questionnaire-based survey, a total of 5,328 patients were included. ${ }^{27}$ Approximately $76 \%$ of all 
patients required not more than 9 minutes to be trained by a nurse to use the prefilled follitropin alfa pen. Patients reported that, on average, they had to change 2.3-2.4 times per treatment cycle. A great majority of the patients having experience with both pens rated the use $(62 \%)$ and the daily injection $(60 \%)$ as being easier with the prefilled follitropin alfa pen as compared to other existing injection methods. When asked to evaluate the use of the prefilled follitropin alfa pen compared with the reusable follitropin beta pen, $30.2 \%$ respondents gave a score of 10 on a scale of $1-10(1=$ less easy and $10=$ much more easy). Approximately $84 \%$ of the patients preferred the prefilled follitropin alfa pen, $9 \%$ the reusable follitropin beta pen, $6 \%$ vials and syringes, and $1 \%$ ampoules and syringes for the injection of r-hFSH.

In two recent studies, a redesigned follitropin alfa pen injector was studied, first as a market research study in a more theoretical way and then under clinical conditions. This third-generation of prefilled follitropin alfa self-injection pen has new and improved features in order to offer an enhanced patient-friendly use. In the second-generation pen, a visually improved numbering on the dose-setting dial was included. This redesigned pen now shows a dose-display window with a magnifying glass lid to enlarge the dosing number, which shows only the selected preset dose, the return of the dose reading to zero after injection of the full prescribed dose or information on how much residual dose is required with a second pen if the full dose has not been administered, and a fully transparent cartridge container with graduated markings to assist the user in determining the approximate amount of product left in the pen. Seventy-three women with some experience with self-injectable gonadotropins and 28 nurses had to simulate injections (water in an orange) in order to learn to handle this new device. Immediately after this, they completed questionnaires to evaluate their experiences. Approximately $88 \%$ of the women attested the use as easy to learn, $66 \%$ of the treatment-experienced patients confirmed the improvements of the redesigned device, and $70 \%$ expressed their wish to use such a pen for all injectable fertility medications. Most of the nurses (96\%) declared that compared with other devices it was much easier, or at least as easy, to teach the patients the use of this new pen. Based on ease of teaching, $68 \%$ of the nurses, especially those who had earlier preferred the follitropin $\beta$ pen, would choose to teach the redesigned pen in preference to any other pen or syringe/vial currently available. ${ }^{28}$

In one observational Australian study, patients evaluated various aspects relating to the ease of use of the redesigned follitropin alfa injection pen by completing two questionnaires, which were completely filled by 72 of the 86 patients included in the study. Also, 26 nurses participated at this study. Approximately $92 \%$ of the patients and also of the nurses found the pen easy to use. $81.4 \%$ of the patients were confident about using the pen correctly and $84.6 \%$ of the nurses found that with these improved features much less or less potential for errors during setting the dose exists. ${ }^{29}$

\section{Summary and conclusion}

The follitropin alfa (filled by mass $[\mathrm{FbM}]$ ) prefilled pen creates a higher level of confidence through its ease of handling in terms of dose preparation and lower potential for making errors compared to other injection procedures. It represents an accurate, ready-to-use device, which is an easy, simple, and nearly pain-free method for the delivery of gonadotrophins in COS for assisted reproductive technologies. It allows precise and individualized dosing. The third-generation of the follitropin alfa pen now dispense over dose increments of $12.5 \mathrm{IU}$ and total dosages ranging from 12.5 to $450 \mathrm{IU}$ in the two greater sizes. ${ }^{30}$ With three prefilled sizes available, 300,450 , and $900 \mathrm{IU}$ in a concentration of $44 \mu \mathrm{g} / \mathrm{mL}$, a personalized prescription is very easy to realize and the small increments allow exact individual COS with r-hFSH. The batch-to-batch consistency with the very small variance of only $\pm 2 \%$ compared with the $45 \%$ variance in not filled by mass gonadotropins allows for accurate and reliable dosing, yielding a more predictable ovarian response. In order to avoid cycle cancellation because of OHSS on one side or nonresponse or too poor response on the other side, more and more individualized COS according to testing the ovarian reserve is performed. The "one size fits all" attitude contains too much risk for the treated women. Such individualization of FSH dose is also likely to be cost-effective in women who are eligible for IVF treatment. ${ }^{31}$

The more accurate and easy-to-use the devices in assisted reproductive techniques the more compliant patients are in administering the correct dose. Patients' verdict is that it is easy to use and thereby errors are reduced. Available alternatives such as monodose or multidose vials in which the lyophilized powdered medication is mixed with a diluent by the patients themselves results in a much greater risk of preparation mistakes and contamination. Furthermore, an individualized COS with such small increments, absolutely necessary in special patients like as patients with, for example, severe polycystic ovary syndrome, is not possible with these devices of powered medication and enclosed solvent, even if specifically marked syringes (IU and not as 
usually $\mathrm{mL}$ ) are used. The risk that the right dosage is not injected is quite great.

The follitropin alfa $(\mathrm{FbM})$ prefilled pen is a clear movement toward better quality of treatment and also better quality of life for the women during this stressful period. Patients are more confident that the right dose has been administered. Accurate dose delivery is very important, and the third-generation of pens dispose over a graduated reservoir holder to allow the patient to control how many units were injected and the residual quantity. ${ }^{30}$ Patients prefer the prefilled pen over ampoules/vials with conventional syringes and the necessity of mixing with a diluent or a reusable pen with cartridges. It has been shown in a recent study from Sweden that patients are willing to pay several hundred Euros to administer the FSH using a prefilled injection pen instead of a conventional syringe and for saving time during the application procedure. They are willing to pay more to receive the recombinant gonadotropin instead of extract from urine from postmenopausal women even if highly purified and for reducing the dose variability. ${ }^{32}$

We can conclude that patients have a preference for modern treatment. All studies showed a high level of patient satisfaction with the prefilled follitropin alfa pen for daily self-administration of r-hFSH.

\section{Disclosure}

The author reports no conflicts of interest in this work.

\section{References}

1. World Health Organization. Reproductive Health Indicators Reproductive Health and Research Guidelines for Their Generation, Interpretation and Analysis for Global Monitoring. Geneva, Switzerland: WHO; 2006.

2. Chandra A, Copen CE, Stephen EH. Infertility and Impaired Fecundity in the United States, 1982-2010: Data from the National Survey of Family Growth. National Health Statistics Reports; no 67. Hyattsville, MD: National Center for Health Statistics; 2013.

3. Bühler K, Bals-Pratsch M, Blumenauer V, et al. DIR annual 2011 - German IVF-registry. J Reproduktionsmed Endokrinol. 2012;9:456-484.

4. Mascarenhas MN, Flaxman SR, Boerma T, Vanderpoel S, Stevens GA. National, regional, and global trends in infertility prevalence since 1990: a systematic analysis of 277 health surveys. PLoS Med. 2002;9(12): e1001356.

5. Department of Health. Regulated Fertility Services: A Commissioning Aid. June 2009. Available from: http://webarchive.nationalarchives.gov. uk/20130107105354/http://www.dh.gov.uk/prod_consum_dh/groups/ dh_digitalassets/documents/digitalasset/dh_101068.pdf. Accessed April 4, 2015.

6. Lunenfeld B. Historical perspectives in gonadotrophin therapy. Hum Reprod Update. 2004;10:453-467.

7. Donini P, Montezemolo R. Rassegna di Clinica, Terapia e Scienze Affini. A publication of the Biologic Laboratories of the Instituto Serono. 1949;48:3-28.

8. Lunenfeld B. Treatment of anovulation by human gonadotropins. Int J Obstet Gynecol. 1963;1:153-157.
9. van de Weijer BH, Mulders JW, Bos ES, Verhaert PD, van den Hooven HW. Compositional analyses of a human menopausal gonadotrophin preparation extracted from urine (menotropin). Identification of some of its major impurities. Reprod Biomed Online. 2003;7: $547-557$.

10. Van Dorsselaer A, Carapito C, Delalande F, et al. Detection of prion protein in urine-derived injectable fertility products by a targeted proteomic approach. PLoS One. 2011;6:e17815.

11. Committee on Safety of Medicines. Scientific Committee on Medicinal Products and Medical Devices Opinion on the Risk Quantification for CJD Transmission via Substances of Human Origin. 1998. Available from: http://ec.europa.eu/health/archive/ph_risk/committees/scmp/ documents/out12_en.pdf. Accessed April 4, 2015.

12. Howles CM, Ezcurra D, Homburg R. Ovarian stimulation protocols in assisted reproductive technology: an update. Expert Rev Endocrinol Metab. 2012;7:319-330.

13. Lehert P, Schertz JC, Ezcurra D. Recombinant human folliclestimulating hormone produces more oocytes with a lower total dose per cycle in assisted reproductive technologies compared with highly purified human menopausal gonadotrophin: a meta-analysis. Reprod Biol Endocrinol. 2010;8:112-123.

14. Yearbooks of the German IVF Registry DIR 1996-2002. Available from: http://www.deutsches-ivf-register.de/jahrbuch-archiv.php. Accessed April 30, 2015.

15. van Wely M, Kwan I, Burt AL, et al. Recombinant versus urinary gonadotrophin for ovarian stimulation in assisted reproductive technology cycles [review]. Cochrane Database Syst Rev. 2011; 16(2):CD005354.

16. Howles CM. Role of LH and FSH in ovarian function. Mol Cell Endocrinol. 2000;161:25-30.

17. Bassett R, Lispi M, Ceccarelli D, et al. Analytical identification of additional impurities in urinary-derived gonadotrophins. Reprod Biomed Online. 2009;19:300-313.

18. Ludwig M, Rabe T, Bühler K, Diedrich K, Felberbaum R. Efficacy of recombinant human FSH in comparison to urinary hMG following a long Downregulation protocol - an analysis of 24764 ART cycles in Germany. J Reproduktionsmed Endokrinol. 2004;1:284-288.

19. Driebergen R, Baer G. Quantification of follicle stimulating hormone (follitropin alfa): is in vivo bioassay still relevant in the recombinant age? Curr Med Res Opin. 2003;19:41-46.

20. Hugues JN, Barlow DH, Rosenwaks Z, et al. Improvement in consistency of response to ovarian stimulation with recombinant human follicle hormone resulting from a new method for calibrating the therapeutic preparation. Reprod Biomed Online. 2003;6:185-190.

21. Wikland M, Hugues JN, Howles C. Improving the consistency of ovarian stimulation: follitropin alfa filled-by-mass. Reprod Biol Endocrinol. 2006; 12:663-668.

22. Tredway D, Fiszbajn G, Yeko T. Improvement of clinical profile with Gonal-f Filled-by-Mass in ovulation induction (preliminary results from 1st treatment cycle). Presented at: VIIth FLASEF Congress, Montevideo, Uruguay, November 18-21, 2002, F54.

23. Bustillo M. Unsuccessful oocyte retrieval: technical artefact or genuine 'empty follicle syndrome'? Reprod Biomed Online. 2004;8:59-67.

24. Markle RL, King PJ, Martin DP, Kutteh WH, Ke RW. Characteristics of successful human chorionic gonadotropin (hCG) administration in assisted reproduction. Fertil Steril. 2002;78(Suppl 1):71-72.

25. Platteau P, Laurent E, Albano C, et al. An open, randomized singlecentre study to compare the efficacy and convenience of follitropin $\beta$ administered by a pen device with follitropin $\alpha$ administered by a conventional syringe in women undergoing ovarian stimulation for IVF/ICSI. Hum Reprod. 2003;18:1200-1204.

26. Porter R, Kissel C, Saunders H, Keck C. Patient and nurse evaluation of recombinant human follicle-stimulating hormone administration methods: comparison of two follitropin injection pens. Curr Med Res Opin. 2008;24:727-735. 
27. Welcker JW, Nawroth F, Bilger W. Patient evaluation of the use of follitropin alfa in a prefilled ready-to-use injection pen in assisted reproductive technology: an observational study. Reprod Biol Endocrinol. 2010; 8:111-117.

28. Abbotts C, Salgado-Braga C, Audibert-Gros C. A redesigned follitropin alfa pen injector for infertility: results of a market research study. Patient Prefer Adherence. 2011;5:315-331.

29. Illingworth PJ, Lahoud R, Quinn F, et al. Single-arm, observational study of the ease of use of a redesigned pen device to deliver recombinant human follicle-stimulating hormone (follitropin alfa) for assisted reproductive technology treatment. Patient Prefer Adherence. 2014;8:813-826.
30. Christen M, Schertz JC, Arriagada P, Keitel J, Müller H. The redesigned follitropin a pen injector for infertility treatment. Expert Opin Drug Deliv. 2011;8:833-839.

31. Moolenaar LM, Broekmans FJ, van Disseldorp J, et al. Cost effectiveness of ovarian reserve testing in in vitro fertilization: a Markov decision-analytic model. Fertil Steril. 2011;96:889-864.

32. Landfeldt E, Jablonowska B, Norlander B, et al. Patient preferences for characteristics differentiating ovarian stimulation treatments. Hum Reprod. 2012;27:760-769.

33. DIR Deutsches IVF Register [homepage on the Internet]. Available from: www.deutsches-ivf-register.de. Accessed April 4, 2015. German.

\section{Publish your work in this journal}

Therapeutics and Clinical Risk Management is an international, peerreviewed journal of clinical therapeutics and risk management, focusing on concise rapid reporting of clinical studies in all therapeutic areas outcomes, safety, and programs for the effective, safe, and sustained use of medicines. This journal is indexed on PubMed Central, CAS,
EMBase, Scopus and the Elsevier Bibliographic databases. The manuscript management system is completely online and includes a very quick and fair peer-review system, which is all easy to use. Visit http://www.dovepress.com/testimonials.php to read real quotes from published authors.

Submit your manuscript here: http://www.dovepress.com/therapeutics-and-clinical-risk-management-journal 